\section{G295(P) DEVELOPMENTAL DELAY IN CHRONIC KIDNEY DISEASE - SERVICE EVALUATION}

M Negoita, P Yadav. Paediatric Nephrology, Leeds Teaching Hospitals NHS Trust, Leeds, UK

10.1136/archdischild-2020-rcpch.256

Background Chronic kidney disease (CKD) is strongly associated with developmental delay (DD). The underlying condition leading to CKD may include $\mathrm{DD}$ as the usual presentation and/or disease progression (e.g. VACTERL, congenital nephrotic syndrome). Also, children with CKD require increased levels of medical intervention with significant associated cardiovascular, haematological and gastrointestinal co-morbidities. Growth can be impaired due to CKD, with a secondary effect on the child's ability for normal development.

Aims To analyse whether children with CKD seen in our tertiary paediatric nephrology clinics have associated DD. Is developmental progression being considered during reviews? And what is being done when a delay is identified?

Methods Twenty CKD patients from our database were randomly chosen for analysis. Paper and electronic records were analysed, using a standardized data collection form. Information collected included: demographics, age when first known to our services, underlying diagnosis, identified DD and referrals made, history of renal transplant, social and safeguarding concerns contributing to difficulties.

Results Age range at time of project: 12 months -6 years. Most patients $(\mathrm{n}=14)$ had first appointment with paediatric nephrology within 4 weeks of life. Most had congenital nephrotic syndrome $(n=5)$, renal dysplasia $(n=5)$ or posterior urethral valves $(\mathrm{n}=3)$. VACTERL association, Lowe syndrome, nephrogenic Diabetes insipidus, Wilms tumour, atypical Haemolytic-uraemic syndrome and prematurity with early bowel surgery were also present. Four children had received a kidney transplant. Eight children had DD identified early. Eight patients had neurodevelopmental and six had general paediatric follow up. Social issues were identified in 4 cases and 6 had safeguarding concerns. Two children were removed from biological parents at birth.

Conclusions DD is common in CKD, especially at the severe end of the spectrum, therefore developmental progression should be part of the general assessment of children with CKD. Timely referral to neurodevelopmental paediatrics is strongly advised, if a form of delay is expected as part of their underlying condition or identified along the way. Because the sooner we start supporting these children in the community, in education, the better for their long-term prognosis.

A referral pathway was developed to aid timely referral to neurodevelopmental services in our region.

\section{G296(P) OUTCOME OF NNON-INVASIVE VENTILATION (NIV) USED IN PICU PATIENTS}

A Nasim, L Verhulst, A Bedford. PICU, Kings College Hospital NHS Trust, London, UK

\subsection{6/archdischild-2020-rcpch.257}

Retrospective study Paediatric Intensive Care Unit in a tertiary care hospital.

54 Patient's data reviewed between April 2016 to March 2018 .
Data was collected from Electronic patient record (EPR) and Metavision and reviewed for different variables as below. Results Most of the children who received NIV were between $0-1$ year of age which make up $53.7 \%$ of the all who received NIV. Children between 2-5 years make up 24.1\%. Percentage of males and females was 66.7 and 33.3 respectively. $37 \%$ of the children's weight was between $5-10 \mathrm{~kg}$ and $25.9 \%$ were $2-5 \mathrm{~kg}$.Major diagnosis was bronchiolitis. Other major diagnosis was LRTI both made up 38.9\% and $27.8 \%$ respectively. Total $21 / 54$ i.e. $38.8 \%$ of the patients had different comorbidities like Genetic problems, metabolic problems, Head injury, neuromuscular problems etc.

$34 / 54(62.9 \%)$ patients were stepped down from mechanical ventilation to NIV. 34/54 (62.9\%) patients were escalated from High flow nasal cannula to NIV.

Nasal mask was used in $46 \%$ of cases. Face mask was used in $18.5 \%$ of the cases.

While using CPAP, $73 \%$ of time the fio $2 \%$ used was less than $50 \%$ and only $5.5 \%$-time fio 2 was more than $70 \%$. 54\% of time the peep used was $8-10 \mathrm{~cm}$ of $\mathrm{H} 20$ and $43 \%$ it was between 5 and 7. When BIPAP was used, EPAP used was 5-6 for $56 \%$ and IPAP was used between 18-20 for 63\% cases.

In $1 / 3$ cases NIV was used for less than 24 hours. In another $1 / 3$ rd NIV was used for up to 3 days. Significant improvement in clinical observations (Especially HR \& RR) were noted after starting on NIV.

In $10 \%$ of the cases contraindications were present, a degree of upper airway obstruction and a reduced level of consciousness.

Most patients (92.6\%) were successfully weaned from NIV. Only 7.4 percent required escalation of support to mechanical ventilation.

1 patient on NIV died as a consequence of a brain tumour.

Conclusion(s) NIV use was effective in avoiding invasive mechanical ventilation in a significant number of patients/inpatients admitted in PICU. NIV was successfully used in patients after stepping down from invasive mechanical ventilation. Close monitoring was the key to the success. Apart from skin damage, no other complications were documented.

\section{G297(P) HYPERTONIC SALINE- AN OPTION FOR TREATMENT OF BRONCHIOLITIS-A LITERATURE REVIEW}

M Kamal, A Umar. Tallaght University Hospital, AMNCH, Dublin, Ireland

\subsection{6/archdischild-2020-rcpch.258}

Bronchiolitis is most common lower respiratory tract infection in children up to 2 years of age. It is most common during the winter months and leading cause of hospitalization of children worldwide. Bronchiolitis is caused by Respiratory syncytial virus (RSV) most of the time but there are other pathogens including adenovirus, rhino/entero virus etc. The current management is minimal handling, oxygen supplementation and NG feeding/intravenous fluids. Many studies and randomized control trials(RCTs) have been done and many are still going on for many years to find other medical solutions for the cure of bronchiolitis. Nebulized hypertonic saline(HS) is one of them which has been under many trials.

The aim of this study was to review the current knowledge of HS regarding its efficacy, safety, clinical effects and 
tolerance in children. We performed a systematic search of the databases PubMed, Pediatrics Pulmonology, European journal of paediatrics, chest physician and Cochrane review for last 5 years.

Method Thirteen clinical studies and Randomized control trials which were done on children treated with hypertonic saline with bronchiolitis hospitalised in an emergency department and general ward were included from year 2014-2019. We included 8 RCT, 1 meta-analysis, 1 Cochrane or 1 decision analysis and 2 Trial sequential analysis and excluded the studies/trials included in Cochrane review 2017 and Meta-analysis 2018. Main outcome of 8 trial out of these 13 trials was 'Length of stay(LOS)' in hospital and 'admission rate' was studied in 1 of these. 1 trial used 'fit for discharge' as main outcome in their trial and 2 Trials looked at the cost effectiveness of use of $\mathrm{HS}$ in bronchiolitis.

Results The available studies suggest that Hypertonic saline is promising and safe to use in children with bronchiolitis but there is no superiority found when compared with NS or standard care in reducing the length of hospital stay or admission from emergency department.

Conclusion Hypertonic saline is safe to use in children with bronchiolitis but there is no extra benefit of it in reducing the disease severity, admissions rate or length of stay.

\section{G298(P) DIAGNOSTIC DILEMMA: RESPIRATORY DISTRESS IN A PRE-TERM WITH SITUS INVERSUS TOTALIS}

MN Naseem, MA Alam. Newham Neonatal Unit, Barts Health NHS Trust, London, UK

\subsection{6/archdischild-2020-rcpch.259}

Introduction Primary ciliary dyskinesia (PCD) is a rare inherited disease affecting motile cilia lining the respiratory tract. ${ }^{1}$ Despite neonatal respiratory distress as an early feature, diagnosis is typically delayed until late childhood.

Case Summary We herein report a case of a late pre-term neonate born at 35 weeks and 2 days with respiratory distress from birth. The baby had to be intubated for a day, shifted between BiPAP and CPAP over 7 days before weaning down to nasal canula oxygen and eventually self-ventilating in air by 2 weeks. He has situs inversus with dextrocardia (situs inversus totalis). He also had an episode of right lung collapse which re-expanded on its own. There is family history of consanguinity and 2 previous neonatal deaths for which the parents were investigated extensively by clinical geneticist but without any conclusive etiology. Baby is referred to Royal Brompton Hospital to be investigated for primary ciliary dyskinesia in an outpatient setting.

Aim The purpose of this case report is to include the possibility of primary ciliary dyskinesia as an earlier diagnosis to facilitate earlier onset of therapy, which would help reduce longterm pulmonary morbidity and mortality. ${ }^{2}$

Results The patient was diagnosed with primary ciliary dyskinesia with cilia completely static on light microscopy and immunofluorescent antibody staining showed an absence of the outer dynein arms.

Conclusion Primary ciliary dyskinesia should be part of differential diagnosis of a neonate who has respiratory distress syndrome in the neonatal period with background history of situs totalis inversus in order to decrease long term pulmonary morbidity and to increase quality of life for the infant.

\section{REFERENCES}

1. Tara Mullowney, David Manson, Raymond Kim, Derek Stephens, Vibhuti Shah, Sharon Dell: Pediatrics. 2014 Dec; 134(6): 1160-1166. doi: 10.1542/peds.20140808

2. Holzmann, D. \& Felix, H. Eur J Pediatr (2000) 159: 857. https://doi.org/10.1007/ PL00008354

\section{G299(P) ANTIBIOTIC USAGE AND CHEST RADIOGRAPHY IN CHILDREN ADMITTED FOR COMMUNITY ACQUIRED PNEUMONIA}

${ }^{1} \mathrm{AC}$ Round, ${ }^{2} \mathrm{KF} \mathrm{Ng},{ }^{2} \mathrm{D}$ Vasudeva, ${ }^{2} \mathrm{~N}$ Manzoor, ${ }^{2} \mathrm{R}$ Radcliffe, ${ }^{2} \mathrm{D}$ Lo, ${ }^{3} \mathrm{~S}$ Koo. ${ }^{1}$ Leicester Medical School, University of Leicester, Leicester, UK; ${ }^{2}$ Paediatrics, Leicester Royal Infirmary, Leicester, UK; ${ }^{3}$ Microbiology, Leicester Royal Infirmary, Leicester, UK

\subsection{6/archdischild-2020-rcpch.260}

Aims To audit the antibiotic choice, route of administration, and duration of treatment; as well as frequency of, and indication for, chest radiographs among children admitted for CAP.

Methods A retrospective audit was conducted on patients aged 0-18 who were admitted with a diagnosis of CAP between December 2018 and February 2019. Patients were identified by International Statistical Classification of Diseases and Related Health Problems (ICD) discharge coding. Data were collected from 50 patients' case notes and electronic notes.

Results

Chest x-ray: The median number of CXR performed was 1. Performance of more than one CXR occurred in 32\% (16/ $50)$ of children, $31 \%(5 / 16)$ of whom did not require oxygen supplementation or PICU admission.

Antibiotic usage:

Antibiotic choice: Amoxicillin was prescribed as the first line antibiotic for $13 \%(6 / 48)$ of children admitted with CAP. Instead, 56\% (27/50) were started on cefuroxime, 10\% (5/48) co-amoxiclav, 8\% (4/48) ceftriaxone and 13\% (6/48) on other antibiotics. A combination of 2 or more antibiotics were given to $55 \%(26 / 48)$ of patients; $19 \%$ of which had no documented reason found. The decision to prescribe multiple antibiotics was not associated with age, presence of co-morbidity, oxygen requirement, PICU admission, length of hospitalisation or antibiotic duration.

Antibiotic route: IV antibiotics were started in $81 \%(39 / 48)$ of children, with no documented justification of this decision in 26\% (10/39) of cases. The presence of co-morbidity and oxygen requirement were not statistically associated with antibiotic route of administration.

Antibiotic duration: The median duration of antibiotics was 7 days (range 1-67 days).

Conclusions Our audit suggests there was excessive usage of broad-spectrum antibiotics for the treatment of inpatient paediatric CAP; contrary to BTS standards recommending amoxicillin as first line antibiotic choice. Following BTS standards recommending that oral antibiotics are effective even in severe CAP and the lack of association between oxygen requirement and antibiotic route, we suspect that the proportion of children started on oral antibiotic for CAP should have been higher. There is a need to improve CAP management in children, particularly regarding antibiotic choice and route of administration as well as reducing the number of unnecessary CXR performed on children admitted for CAP. 